\title{
Palladium-Catalyzed Allylalkynylation of Benzynes: a Highly Efficient Route to Substituted 1-Allyl-2-alkynylbenzenes
}

\author{
Masilamani Jeganmohan, and Chien-Hong Cheng ${ }^{*}$ \\ Department of Chemistry, Tsing Hua University, Hsinchu, Taiwan 300 \\ chcheng@mx.nthu.edu.tw
}

Supporting Information 


\section{Experimental Section}

General procedure for the allylalkynylation of benzynes 1 with allylic chlorides 2, and alkynylstannanes 3: A 25-ml round-bottom side-arm flask containing $\mathrm{Pd}(\mathrm{dba})_{2} / \mathrm{dppe}(0.0500 \mathrm{mmol}), \mathrm{CsF}(3.00 \mathrm{mmol})$ was evacuated and purged with nitrogen gas three times. To the flask were then added benzyne precursor (1) (1.00 mmol), an allyl chloride (2) (1.20 mmol), alkynylstannane (3) (1.20 mmol) and $\mathrm{CH}_{3} \mathrm{CN}(3.0 \mathrm{ml})$ via syringes. The reaction mixture was allowed to stir at $40{ }^{\circ} \mathrm{C}$ for 10 h. At the end of the reaction, the reaction mixture was diluted with $\mathrm{CH}_{2} \mathrm{Cl}_{2}$, filtered through Celite and silica gel. The filtrate was concentrated and the residue was purified through a silica gel column using hexane as eluent to give pure 4 .

Expremental procedure for the cyclization of 1-(1-hexynyl)-2-(2-methylallyl)benzene (4b) with 1-iodonapthalene. A 10-ml sealed flask containing $\mathrm{Pd}(\mathrm{OAc})_{2}(0.0500 \mathrm{mmol}), \mathrm{PPh}_{3}(0.100 \mathrm{mmol})$, and $\mathrm{Tl}(\mathrm{OAc})$ (1.2 mmol) was evacuated and purged with nitrogen gas three times. To the flask were then added 1-(1-hexynyl)-2-(2-methylallyl)benzene $\quad(\mathbf{4 b}) \quad(1.00 \quad \mathrm{mmol})$, 1-iodonapthalene $(1.00 \mathrm{mmol})$ and DMF $(3.0 \mathrm{ml})$ via syringes. The solution was stirred at $120{ }^{\circ} \mathrm{C}$ for $10 \mathrm{~h}$ and. At the end of the reaction, the reaction mixture was filtered through a Celite and silica gel pad and washed with $50 \mathrm{ml}$ of ether. The filtrate was washed with water, dried with $\mathrm{MgSO}_{4}$ and concentrated in vacuo. The residue was purified through a silica gel column using hexane as eluent to give pure $\mathbf{5}$.

Spectral data of compounds $\mathbf{4 a - p}, 5$ and $\mathbf{1 1}$ or 12, and ${ }^{1} \mathrm{H}$ NMR Spectra of all compounds are listed bellow:

1-(2-Allylphenyl)-1-hexyne (4a): colorless oil; ${ }^{1} \mathrm{H}$ NMR $\left(600 \mathrm{MHz}, \mathrm{CDCl}_{3}\right): \delta 7.40$ (d, $J=7.2 \mathrm{~Hz}, 1 \mathrm{H}), 7.21-7.14(\mathrm{~m}, 3 \mathrm{H}), 6.19-6.01(\mathrm{~m}, 1 \mathrm{H}), 5.11-5.06(\mathrm{~m}, 2 \mathrm{H})$, $3.56(\mathrm{~d}, J=6.6 \mathrm{~Hz}, 2 \mathrm{H}), 2.46(\mathrm{t}, J=6.6 \mathrm{~Hz}, 2 \mathrm{H}), 1.63-1.49(\mathrm{~m}, 4 \mathrm{H}), 0.97(\mathrm{t}, J=$ 
$7.2 \mathrm{~Hz}, 3 \mathrm{H}) ;{ }^{13} \mathrm{C}$ NMR $\left(150 \mathrm{MHz}, \mathrm{CDCl}_{3}\right): \delta 141.68,136.77,132.11,128.57,127.65$, 125.91, 123.48, 115.70, 94.32, 79.03, 38.74, 30.88, 21.99, 19.21, 13.63; HRMS: calcd for $\mathrm{C}_{15} \mathrm{H}_{18}$ 198.1409, found 198.1404.

1-(1-Hexynyl)-2-(2-methylallyl)benzene (4b): colorless oil; ${ }^{1} \mathrm{H}$ NMR $(600 \mathrm{MHz}$, $\left.\mathrm{CDCl}_{3}\right): \delta 7.40(\mathrm{~d}, J=7.2 \mathrm{~Hz}, 1 \mathrm{H}), 7.21-7.13(\mathrm{~m}, 3 \mathrm{H}), 4.82(\mathrm{~s}, 1 \mathrm{H}), 4.67(\mathrm{~s}, 1 \mathrm{H})$, $3.52(\mathrm{~s}, 2 \mathrm{H}), 2.44(\mathrm{t}, J=6.6 \mathrm{~Hz}, 2 \mathrm{H}), 1.73(\mathrm{~s}, 3 \mathrm{H}), 1.62-1.48(\mathrm{~m}, 4 \mathrm{H}), 0.96(\mathrm{t}, J=$ $7.2 \mathrm{~Hz}, 3 \mathrm{H}) ;{ }^{13} \mathrm{C}$ NMR $\left(150 \mathrm{MHz}, \mathrm{CDCl}_{3}\right): \delta 144.72,141.34,132.09,128.97,127.45$, 125.87, 123.98, 111.74, 93.94, 79.27, 42.42, 30.89, 22.31, 21.99, 19.20, 13.62; HRMS: calcd for $\mathrm{C}_{16} \mathrm{H}_{20} 212.1565$, found 212.1566 .

1-(1-Hexynyl)-2-[(E)-1-methyl-2-butenyl]benzene (4c): colorless oil; ${ }^{1} \mathrm{H}$ NMR (400MHz, $\left.\mathrm{CDCl}_{3}\right): \delta 7.41(\mathrm{~d}, J=7.0 \mathrm{~Hz}, 1 \mathrm{H}), 7.22$ - $7.13(\mathrm{~m}, 3 \mathrm{H}), 5.68-5.62(\mathrm{~m}, 1$ H), $5.49-5.41(\mathrm{~m}, 1 \mathrm{H}), 3.77-3.74(\mathrm{~m}, 1 \mathrm{H}), 2.40(\mathrm{t}, J=6.4 \mathrm{~Hz}, 2 \mathrm{H}), 1.77(\mathrm{~d}, J=$ $6.8 \mathrm{~Hz}, 3 \mathrm{H}), 1.71-1.56(\mathrm{~m}, 4 \mathrm{H}), 1.35(\mathrm{~d}, J=7.6 \mathrm{~Hz}, 3 \mathrm{H}), 0.95(\mathrm{t}, J=7.2 \mathrm{~Hz}, 3 \mathrm{H})$; ${ }^{13} \mathrm{C}$ NMR $\left(100 \mathrm{MHz}, \mathrm{CDCl}_{3}\right): \delta 142.75,135.45,131.21,127.71,127.08,126.78$, 126.09, 124.07, 93.71, 78.82, 42.45, 30.92, 22.37, 21.96, 20.86, 17.26, 13.63; HRMS: calcd for $\mathrm{C}_{17} \mathrm{H}_{22} 226.1721$, found 226.1718 .

1-[2-(2-Cyclohexenyl)phenyl]-1-hexyne (4d): colorless oil; ${ }^{1} \mathrm{H}$ NMR $(600 \mathrm{MHz}$, $\left.\mathrm{CDCl}_{3}\right): \delta 7.36(\mathrm{~d}, J=7.2 \mathrm{~Hz}, 1 \mathrm{H}), 7.20-7.09(\mathrm{~m}, 3 \mathrm{H}), 5.92-5.89(\mathrm{~m}, 1 \mathrm{H}), 5.69-$ $5.67(\mathrm{~m}, 1 \mathrm{H}), 3.94-3.92(\mathrm{~m}, 1 \mathrm{H}), 2.43(\mathrm{t}, J=6.6 \mathrm{~Hz}, 2 \mathrm{H}), 2.08-2.06(\mathrm{~m}, 3 \mathrm{H})$, $1.60-1.48(\mathrm{~m}, 8 \mathrm{H}), 0.94(\mathrm{t}, J=7.2 \mathrm{~Hz}, 3 \mathrm{H}) ;{ }^{13} \mathrm{C} \mathrm{NMR}\left(150 \mathrm{MHz}, \mathrm{CDCl}_{3}\right): \delta 147.84$, $132.06,130.00,128.51,127.13,127.48,125.62,123.10,94.12,78.99,39.28,30.85$ 25.08, 21.94, 21.03, 19.20, 13.61; HRMS: calcd for $\mathrm{C}_{18} \mathrm{H}_{22}$ 238.1721, found 238.1723 .

1-(2-Phenyl-1-ethynyl)-2-[(E)-3-phenyl-2-propenyl]benzene (4e): colorless oil; ${ }^{1} \mathrm{H}$ NMR (400MHz, $\left.\mathrm{CDCl}_{3}\right): \delta 7.41-7.22(\mathrm{~m}, 14 \mathrm{H}), 6.56-6.39(\mathrm{~m}, 2 \mathrm{H}), 3.80(\mathrm{~d}, J=$ $6.6 \mathrm{~Hz}, 2 \mathrm{H}) ;{ }^{13} \mathrm{C}$ NMR $\left(100 \mathrm{MHz}, \mathrm{CDCl}_{3}\right): \delta 139.31,137.41,136.57,132.09,131.04$, 
$130.04,129.73,128.64,128.45,127.60,127.08,126.09,126.73,125.69,92.86,79.11$, 36.63; HRMS: calcd for $\mathrm{C}_{23} \mathrm{H}_{18}$ 294.1409, found 294.1413.

1-(1-Hexynyl)-4,5-dimethyl-2-(2-methylallyl)benzene (4f): colorless oil; ${ }^{1} \mathrm{H}$ NMR (600MHz, $\left.\mathrm{CDCl}_{3}\right): \delta 7.18(\mathrm{~s}, 1 \mathrm{H}), 6.95(\mathrm{~s}, 1 \mathrm{H}), 4.80(\mathrm{~s}, 1 \mathrm{H}), 4.69(\mathrm{~s}, 1 \mathrm{H}), 3.45(\mathrm{~s}, 2$ H), $2.44(\mathrm{t}, J=6.6 \mathrm{~Hz}, 2 \mathrm{H}), 2.22(\mathrm{~s}, 3 \mathrm{H}), 2.20(\mathrm{~s}, 3 \mathrm{H}), 1.73(\mathrm{~s}, 3 \mathrm{H}), 1.61-1.48(\mathrm{~m}, 4$ $\mathrm{H}), 0.96(\mathrm{t}, J=7.2 \mathrm{~Hz}, 3 \mathrm{H}) ;{ }^{13} \mathrm{C} \mathrm{NMR}\left(150 \mathrm{MHz}, \mathrm{CDCl}_{3}\right): \delta 145.15,138.66,136.20$, $134.06,132.98,130.23,121.14,111.37,92.70,79.38,41.97,30.97,22.26,21.97$, 19.63, 19.19, 19.05, 13.62; HRMS: calcd for $\mathrm{C}_{18} \mathrm{H}_{24} 240.1878$, found 240.1881.

5-(1-Hexynyl)-6-(2-methylallyl)indane (4g): colorless oil; ${ }^{1} \mathrm{H}$ NMR $(600 \mathrm{MHz}$, $\left.\mathrm{CDCl}_{3}\right): \delta 7.27(\mathrm{~s}, 1 \mathrm{H}), 7.06(\mathrm{~s}, 1 \mathrm{H}), 4.81(\mathrm{~s}, 1 \mathrm{H}), 4.69(\mathrm{~s}, 1 \mathrm{H}), 3.50(\mathrm{~s}, 2 \mathrm{H}), 2.88-$ $2.84(\mathrm{~m}, 2 \mathrm{H}), 2.44$ (t, $J=6.6 \mathrm{~Hz}, 2 \mathrm{H}), 2.06-2.04(\mathrm{~m}, 2 \mathrm{H}), 1.74(\mathrm{~s}, 3 \mathrm{H}), 1.61-$ $1.49(\mathrm{~m}, 4 \mathrm{H}), 0.96(\mathrm{t}, J=7.2 \mathrm{~Hz}, 3 \mathrm{H}) ;{ }^{13} \mathrm{C} \mathrm{NMR}\left(150 \mathrm{MHz}, \mathrm{CDCl}_{3}\right): \delta$ 145.25, $144.03,141.83,139.26,127.67,124.81,121.46,111.41,92.55,79.85,42.31,32.75$ 32.26, 30.97, 25.38, 22.28, 21.96, 19.19, 13.62; HRMS: calcd for $\mathrm{C}_{19} \mathrm{H}_{24} 252.1878$, found 252.1880 .

5-(1-Hexynyl)-6-(2-methylallyl)-1,3-benzodioxole (4h): colorless oil; ${ }^{1} \mathrm{H}$ NMR (400MHz, $\left.\mathrm{CDCl}_{3}\right): \delta 6.81(\mathrm{~s}, 1 \mathrm{H}), 6.64(\mathrm{~s}, 1 \mathrm{H}), 5.89(\mathrm{~s}, 2 \mathrm{H}), 4.77(\mathrm{~s}, 1 \mathrm{H}), 4.66(\mathrm{~s}, 1$ H), 3.39 (s, $2 \mathrm{H}), 2.38$ (t, $J=6.6 \mathrm{~Hz}, 2 \mathrm{H}), 1.70(\mathrm{~s}, 3 \mathrm{H}), 1.60-1.49$ (m, $4 \mathrm{H}), 0.93$ (t, $J=7.2 \mathrm{~Hz}, 3 \mathrm{H}) ;{ }^{13} \mathrm{C} \mathrm{NMR}\left(100 \mathrm{MHz}, \mathrm{CDCl}_{3}\right): \delta 147.30,145.56,144.87,136.18$, $116.72,111.69,111.46,109.11,101.03,92.35,79.25,42.31,33.34,22.09,19.16$ 13.94, 13.57; HRMS: calcd for $\mathrm{C}_{17} \mathrm{H}_{20} \mathrm{O}_{2} 256.1463$, found 256.1467.

1-(2-Methylallyl)-2-(1-propynyl)benzene (4i): colorless oil; ${ }^{1} \mathrm{H}$ NMR $(500 \mathrm{MHz}$, $\left.\mathrm{CDCl}_{3}\right): \delta 7.46(\mathrm{~d}, J=7.2 \mathrm{~Hz}, 1 \mathrm{H}), 7.28-7.20(\mathrm{~m}, 3 \mathrm{H}), 4.89(\mathrm{~s}, 1 \mathrm{H}), 4.76(\mathrm{~s}, 1 \mathrm{H})$, $3.58(\mathrm{~s}, 2 \mathrm{H}), 2.14(\mathrm{~s}, 3 \mathrm{H}), 1.79(\mathrm{~s}, 3 \mathrm{H}) ;{ }^{13} \mathrm{C} \mathrm{NMR}\left(125 \mathrm{MHz}, \mathrm{CDCl}_{3}\right): \delta$ 144.70, $141.42,132.08,128.95,127.50,125.88,123.92,111.82,89.34,78.50,42.42,22.25$ 4.36; HRMS: calcd for $\mathrm{C}_{13} \mathrm{H}_{14}$ 170.1096, found 170.1095. 
1-(3-Methoxy-1-propynyl)-2-(2-methylallyl)benzene (4j): colorless oil; ${ }^{1} \mathrm{H}$ NMR $\left(400 \mathrm{MHz}, \mathrm{CDCl}_{3}\right): \delta 7.44(\mathrm{~d}, J=8.0 \mathrm{~Hz}, 1 \mathrm{H}), 7.27-7.14(\mathrm{~m}, 3 \mathrm{H}), 4.81(\mathrm{~s}, 1 \mathrm{H})$, $4.65(\mathrm{~s}, 1 \mathrm{H}), 4.34(\mathrm{~s}, 3 \mathrm{H}), 3.51(\mathrm{~s}, 2 \mathrm{H}), 3.44(\mathrm{~s}, 3 \mathrm{H}), 1.71(\mathrm{~s}, 3 \mathrm{H}) ;{ }^{13} \mathrm{C}$ NMR $(100$ $\left.\mathrm{MHz}, \mathrm{CDCl}_{3}\right): \delta 144.38,141.76,132.39,129.11,128.44,125.98,122.59,112.03$ 88.47, 85.15, 60.42, 57.50, 42.42, 22.31; HRMS: calcd for $\mathrm{C}_{14} \mathrm{H}_{16} \mathrm{O} 200.1201$, found 200.1203 .

1-(2-Methylallyl)-2-(2-phenyl-1-ethynyl)benzene (4k): colorless oil; ${ }^{1} \mathrm{H}$ NMR (600 $\left.\mathrm{MHz}, \mathrm{CDCl}_{3}\right): \delta 7.54-7.52(\mathrm{~m}, 3 \mathrm{H}), 7.35-7.20(\mathrm{~m}, 6 \mathrm{H}), 4.85(\mathrm{~s}, 1 \mathrm{H}), 4.74(\mathrm{~s}, 1 \mathrm{H})$, $3.61(\mathrm{~s}, 2 \mathrm{H}), 1.77(\mathrm{~s}, 3 \mathrm{H}) ;{ }^{13} \mathrm{C} \mathrm{NMR}\left(150 \mathrm{MHz}, \mathrm{CDCl}_{3}\right): \delta 144.48,141.70,132.18$, $131.45,129.17,128.15,126.08,123.52,123.19,112.06,92.97,88.32,42.65,22.36$ HRMS: calcd for $\mathrm{C}_{18} \mathrm{H}_{16} 232.1252$, found 232.1249.

1-[2-(4-Bromophenyl)-1-ethynyl]-2-(2-methylallyl)benzene (4I): colorless oil; ${ }^{1} \mathrm{H}$ NMR (600 MHz, $\left.\mathrm{CDCl}_{3}\right): \delta 7.50-7.23(\mathrm{~m}, 8 \mathrm{H}), 4.82(\mathrm{~s}, 1 \mathrm{H}), 4.69(\mathrm{~s}, 1 \mathrm{H}), 3.59$ (s, $2 \mathrm{H}), 1.75(\mathrm{~s}, 3 \mathrm{H}) ;{ }^{13} \mathrm{C}$ NMR $\left(150 \mathrm{MHz}, \mathrm{CDCl}_{3}\right): \delta 144.36,141.72,132.18,131.78$, $131.45,131.61,129.32,128.59,126.15,122.43,122.36,112.13,91.85,89.45,42.65$ 22.37; HRMS: calcd for $\mathrm{C}_{18} \mathrm{H}_{15} \mathrm{Br} 310.0357$, found 310.0357 .

2-2-[2-(2-Methylallyl)phenyl]-1-ethynylthiophene (4m): colorless oil; ${ }^{1} \mathrm{H}$ NMR $\left(600 \mathrm{MHz}, \mathrm{CDCl}_{3}\right): \delta 7.45(\mathrm{~d}, J=7.2 \mathrm{~Hz}, 1 \mathrm{H}), 7.25-7.16(\mathrm{~m}, 5 \mathrm{H}), 6.97(\mathrm{~d}, J=5.4$ Hz, $1 \mathrm{H}), 4.80$ (s, $1 \mathrm{H}), 4.69$ (s, $1 \mathrm{H}), 3.52$ (s, $2 \mathrm{H}), 1.71$ (s, $3 \mathrm{H}) ;{ }^{13} \mathrm{C} \mathrm{NMR}(150 \mathrm{MHz}$, $\left.\mathrm{CDCl}_{3}\right): \delta 144.38,141.61,131.96,131.55,129.31,128.48,127.13,126.12,123.50$ 122.85, 112.16, 92.02, 86.16, 42.71, 22.35; HRMS: calcd for $\mathrm{C}_{16} \mathrm{H}_{14} \mathrm{~S}$ 238.0816, found 238.0816 .

1-(1-Hexynyl)-4-methyl-2-(2-methylallyl)benzene (4n): colorless oil; ${ }^{1} \mathrm{H}$ NMR $\left(600 \mathrm{MHz}, \mathrm{CDCl}_{3}\right): \delta 7.43(\mathrm{~d}, J=7.2 \mathrm{~Hz}, 1 \mathrm{H}), 7.25-7.16(\mathrm{~m}, 3 \mathrm{H}), 5.38(\mathrm{~s}, 1 \mathrm{H})$, $5.29(\mathrm{~s}, 1 \mathrm{H}), 4.82(\mathrm{~s}, 1 \mathrm{H}), 4.70(\mathrm{~s}, 1 \mathrm{H}), 3.50(\mathrm{~s}, 2 \mathrm{H}), 1.99(\mathrm{~s}, 3 \mathrm{H}), 1.73(\mathrm{~s}, 3 \mathrm{H}) ;{ }^{13} \mathrm{C}$ NMR (150 MHz, $\left.\mathrm{CDCl}_{3}\right): \delta 144.47,141.59,132.13,129.16,128.21,126.97,126.01$, 
123.13, 121.57, 111.96, 94.18, 87.29, 42.53, 23.49, 22.30; HRMS: calcd for $\mathrm{C}_{15} \mathrm{H}_{16}$ 196.1252, found 196.1250 .

1-[2-(1-Cyclohexenyl)-1-ethynyl]-2-(2-methylallyl)benzene (40): colorless oil; ${ }^{1} \mathrm{H}$ NMR (600MHz, $\left.\mathrm{CDCl}_{3}\right): \delta 7.40(\mathrm{~d}, J=7.2 \mathrm{~Hz}, 1 \mathrm{H}), 7.21-7.14(\mathrm{~m}, 3 \mathrm{H}), 6.29(\mathrm{t}, J=$ $2 \mathrm{~Hz}, 1 \mathrm{H}), 4.81(\mathrm{~s}, 1 \mathrm{H}), 4.69(\mathrm{~s}, 1 \mathrm{H}), 3.51(\mathrm{~s}, 2 \mathrm{H}), 2.23-2.13(\mathrm{~m}, 4 \mathrm{H}), 1.72(\mathrm{~s}$, 3H), $1.68-1.60(\mathrm{~m}, 4 \mathrm{H}) ;{ }^{13} \mathrm{C}$ NMR $\left(150 \mathrm{MHz}, \mathrm{CDCl}_{3}\right): \delta 144.61,141.29,134.77$, $131.93,129.02,127.75,125.93,123.61,120.87,111.87,94.89,85.62,42.50,29.22$, 25.72, 22.31, 21.49; HRMS: calcd for $\mathrm{C}_{18} \mathrm{H}_{20} 236.1565$, found 236.1568.

1-(2-Allylphenyl)-1-hexyne (4p): colorless oil; ${ }^{1} \mathrm{H} \mathrm{NMR}\left(400 \mathrm{MHz}, \mathrm{CDCl}_{3}\right): \delta 7.60-$ $7.56(\mathrm{~m}, 3 \mathrm{H}), 7.41-7.27(\mathrm{~m}, 6 \mathrm{H}), 6.19-6.02(\mathrm{~m}, 1 \mathrm{H}), 5.21-5.07(\mathrm{~m}, 2 \mathrm{H}), 3.70(\mathrm{~d}$, $J=6.8 \mathrm{~Hz}, 2 \mathrm{H}) ;{ }^{13} \mathrm{C} \mathrm{NMR}\left(100 \mathrm{MHz}, \mathrm{CDCl}_{3}\right): \delta 141.97,136.52,132.15,131.45$, $128.78,128.50,128.38,128.32,126.09,123.42,122.70,116.01,93.26,88.04,38.83$; HRMS: calcd for $\mathrm{C}_{17} \mathrm{H}_{14}$ 218.1096, found 218.1097.

13-Butyl-7a-methyl-7a,8-dihydro-7H-indeno[2,1-b]phenanthrene (5): colorless oil; ${ }^{1} \mathrm{H}$ NMR (500 MHz, $\left.\mathrm{CDCl}_{3}\right): \delta 8.21(\mathrm{~d}, J=8.0 \mathrm{~Hz}, 1 \mathrm{H}), 7.81(\mathrm{t}, J=8 \mathrm{~Hz}, 1 \mathrm{H}), 7.67$ $(\mathrm{d}, J=7.5 \mathrm{~Hz}, 1 \mathrm{H}), 7.48-7.25(\mathrm{~m}, 7 \mathrm{H}), 3.48-3.42(\mathrm{~m}, 1 \mathrm{H}), 3.27-3.20(\mathrm{~m}, 1 \mathrm{H})$, $3.01-2.88(\mathrm{~m}, 4 \mathrm{H}), 1.27-1.08(\mathrm{~m}, 4 \mathrm{H}), 0.88(\mathrm{~s}, 3 \mathrm{H}), 0.66(\mathrm{t}, J=7.0 \mathrm{~Hz}, 3 \mathrm{H}) ;{ }^{13} \mathrm{C}$ NMR (125 MHz, $\left.\mathrm{CDCl}_{3}\right): \delta 148.73,146.52,139.29,134.49,134.37,133.56,130.75$, $130.45,128.60,127.40,127.04,126.60,126.45,126.08,125.70,125.54,124.81$, $124.35,46.99,44.23,42.89,32.91,30.86,22.64,21.70,13.76$; HRMS: calcd for $\mathrm{C}_{26} \mathrm{H}_{26}$ 338.2034, found 338.2036.

1-[5-Methyl-2-(2-methylallyl)phenyl]-1-hexyne (11 and 12): colorless oil; ${ }^{1} \mathrm{H}$ NMR (600MHz, $\left.\mathrm{CDCl}_{3}\right): \delta 7.30(\mathrm{~d}, J=7.2 \mathrm{~Hz}, 1 \mathrm{H}), 7.23(\mathrm{~s}, 1 \mathrm{H}), 7.08-6.95(\mathrm{~m}, 4 \mathrm{H})$, $4.82-4.80(\mathrm{~m}, 2 \mathrm{H}), 4.70-4.67(\mathrm{~m}, 2 \mathrm{H}), 3.48(\mathrm{~m}, 4 \mathrm{H}), 2.45-2.42$ (s, $6 \mathrm{H}), 1.73-$ $1.51(\mathrm{~m}, 8 \mathrm{H}), 0.97-0.95(\mathrm{~m}, 6 \mathrm{H}) ;{ }^{13} \mathrm{C} \mathrm{NMR}\left(150 \mathrm{MHz}, \mathrm{CDCl}_{3}\right): \delta$ 144.94, 144.88, $141.16,138.32,137.32,135.33,132.57,131.92,129.69,128.87,128.38,126.71$, 
$123.73,120.99,111.60,111.50,93.48,93.05,79.42,79.32,42.37,42.01,30.95,30.92$, 22.29, 22.26, 21.98, 21.33, 20.72, 19.19, 13.60; HRMS: calcd for $\mathrm{C}_{17} \mathrm{H}_{22} 226.1721$, found 226.1719 . 

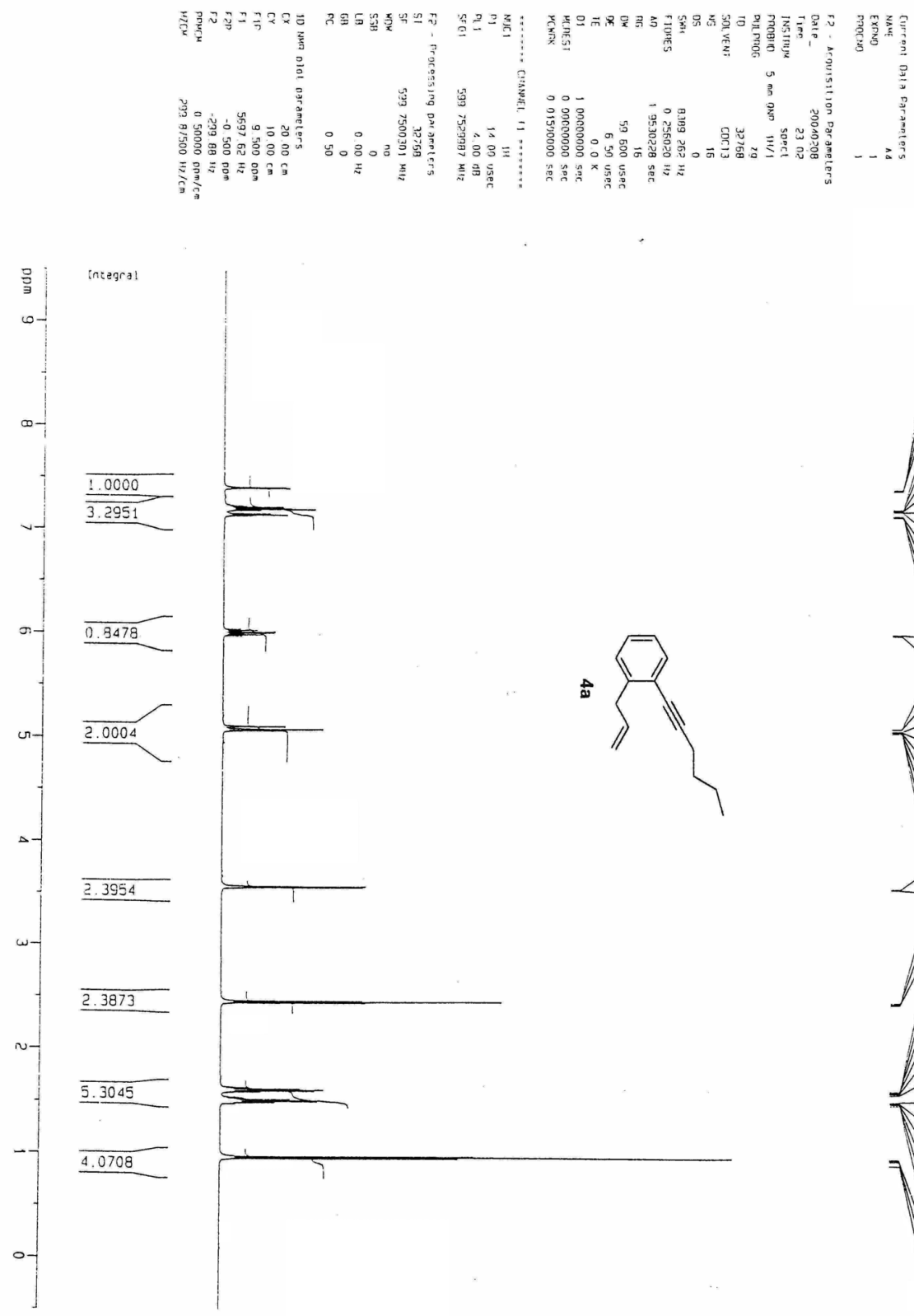

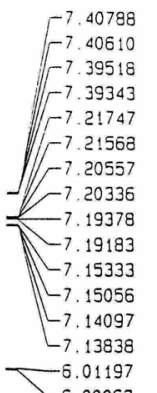
$\Gamma^{5.11101}$ $-5.08665$ $-5.08474$ $-5.08474$ $-5.08252$ $-5.07960$ $-5.06793$ $L_{5} .06484$ $-3.57107$ 3.55989 $r^{2.46961}$ $-34508$ $-1.52519$ $\Gamma^{1.62619}$ $\Gamma^{1.61451}$ $-1.60985$ $-1.60119$ L -1.58926 1.52651
-1.51750 -1.51750
-1.51381 - -1.50527 $L_{-1.50104}$ $\| L_{1.48905}$ $\left[\begin{array}{l}-1.48905 \\ -0.97756\end{array}\right.$ Lo. 96529 $L_{0.95309}$ 

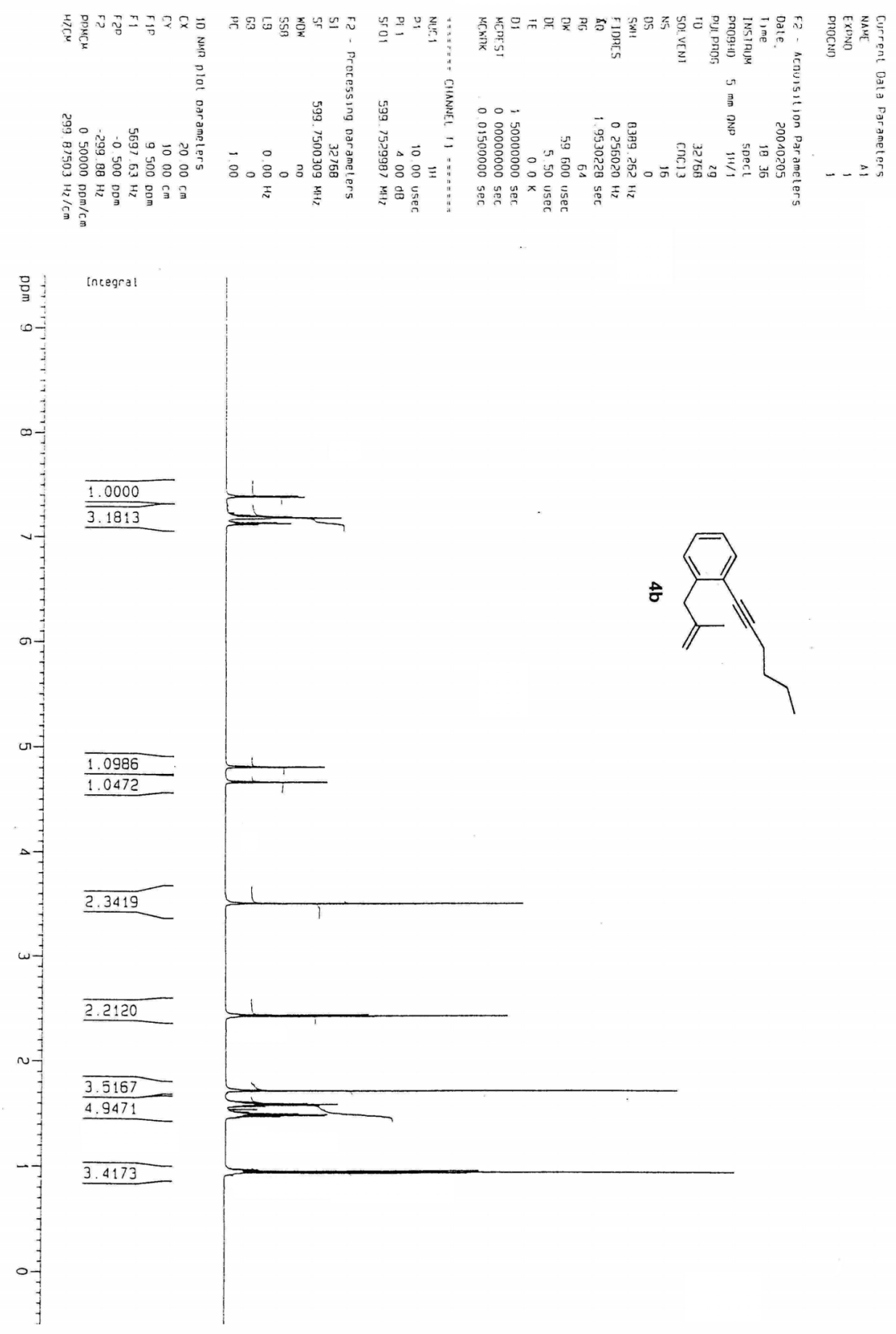

oom

$[7.40484$

7.39233
-7.21135

$-7.20939$

$-7.20004$

$\sqrt{7.19393}$

$-7.18115$

-7.18115
-7.15110

$-7.14806$

$-7.13859$

$-7.12692$

4.82454

$-4.82225$

$-4.82105$

$-4.82020$

$-4.81887$

4.67823
-4.87733

$-4.67626$

$\Gamma^{2 .} 45984$

$\Gamma^{2.45984}$

$-2.43635$

$-1.73259$

$\Gamma^{-1.61650}$

] $r_{-1.60448}^{-1.60093}$

$\left[\Gamma_{-1.59229}^{1.60093}\right.$

$-1.59170$

-1.52030

$-1.50783$

1.49484
-1.48289

$-1.48289$

7

$L_{0.97274}$

$-0.96052$

$-0.94830$ 


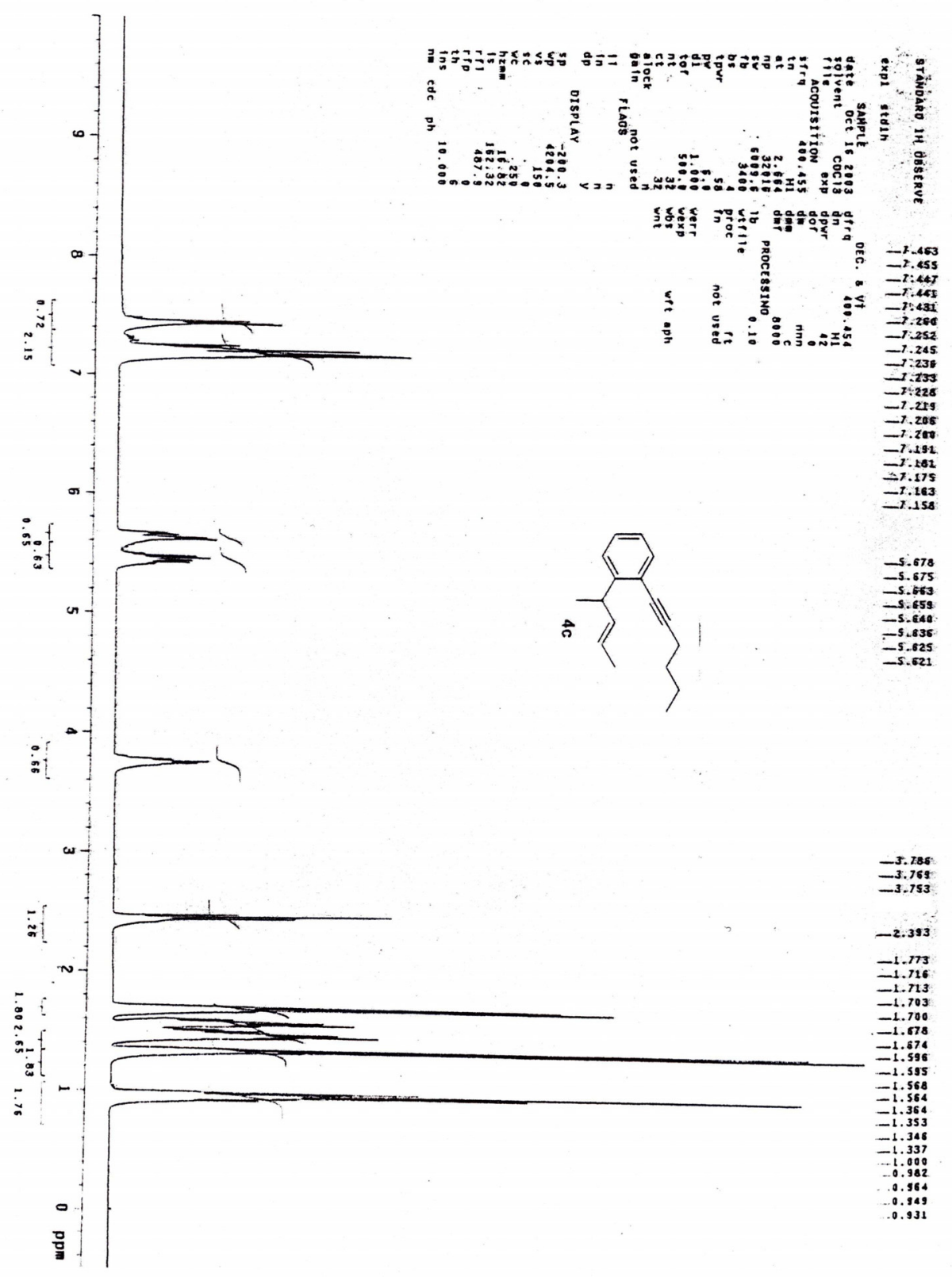



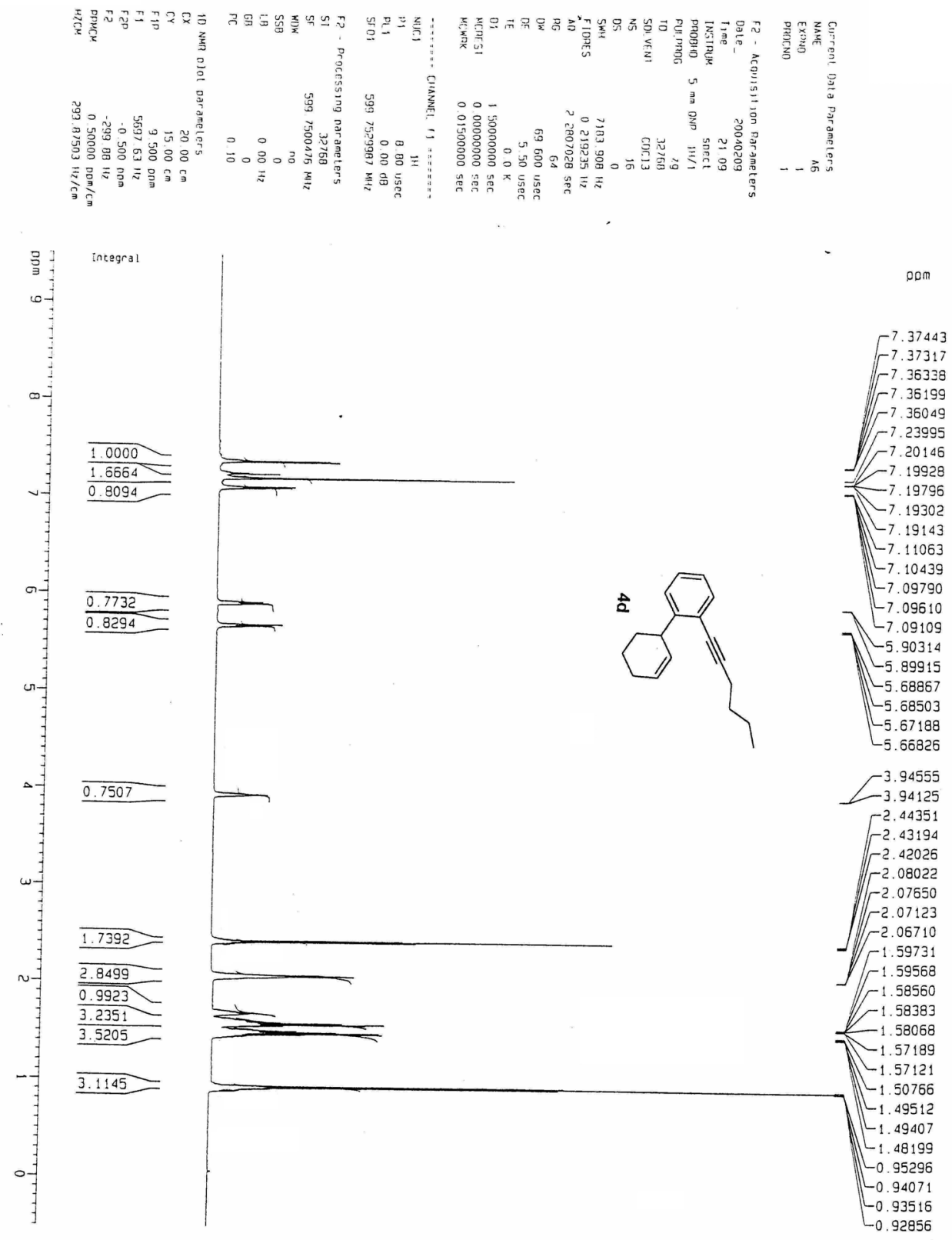


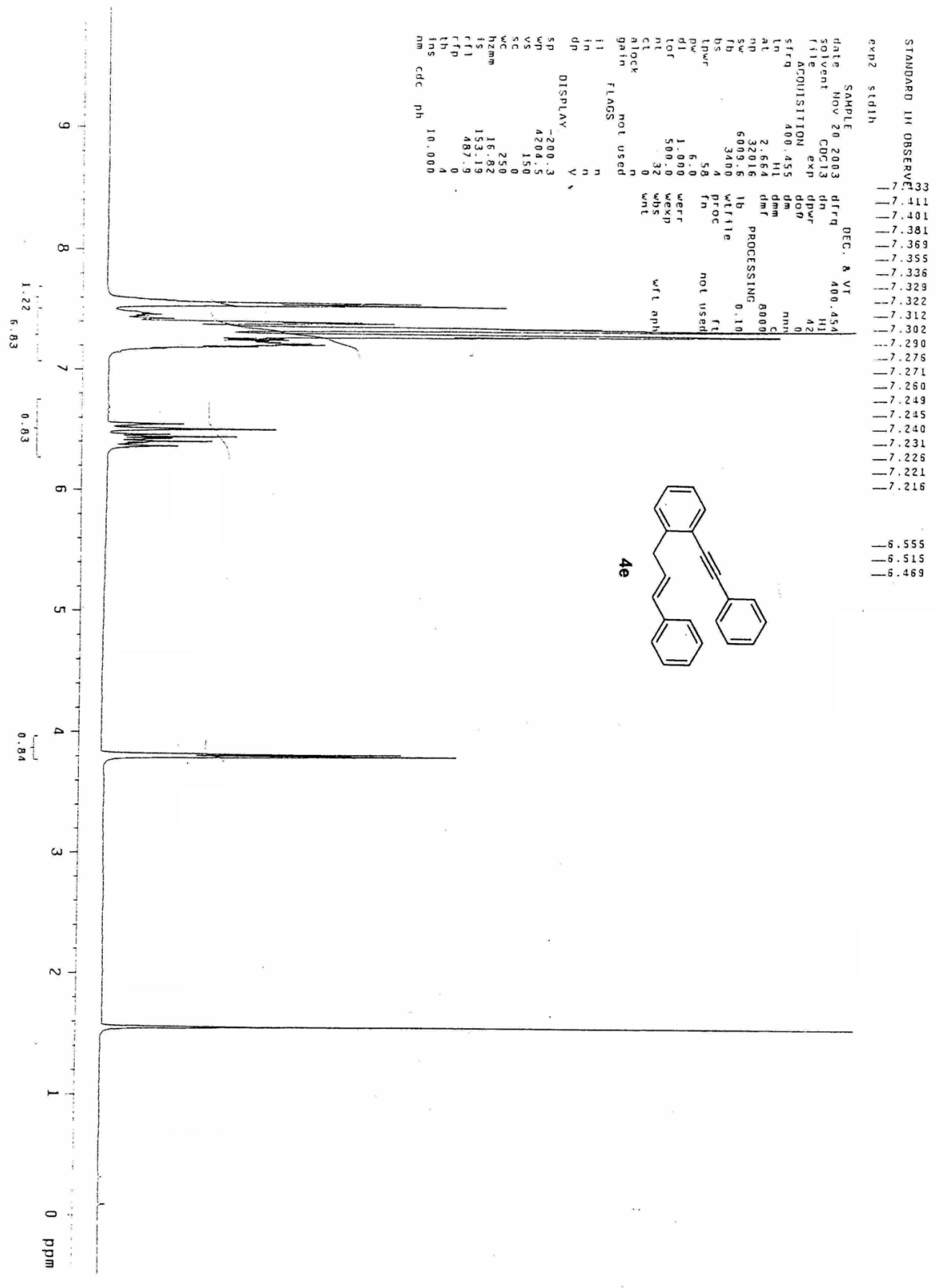



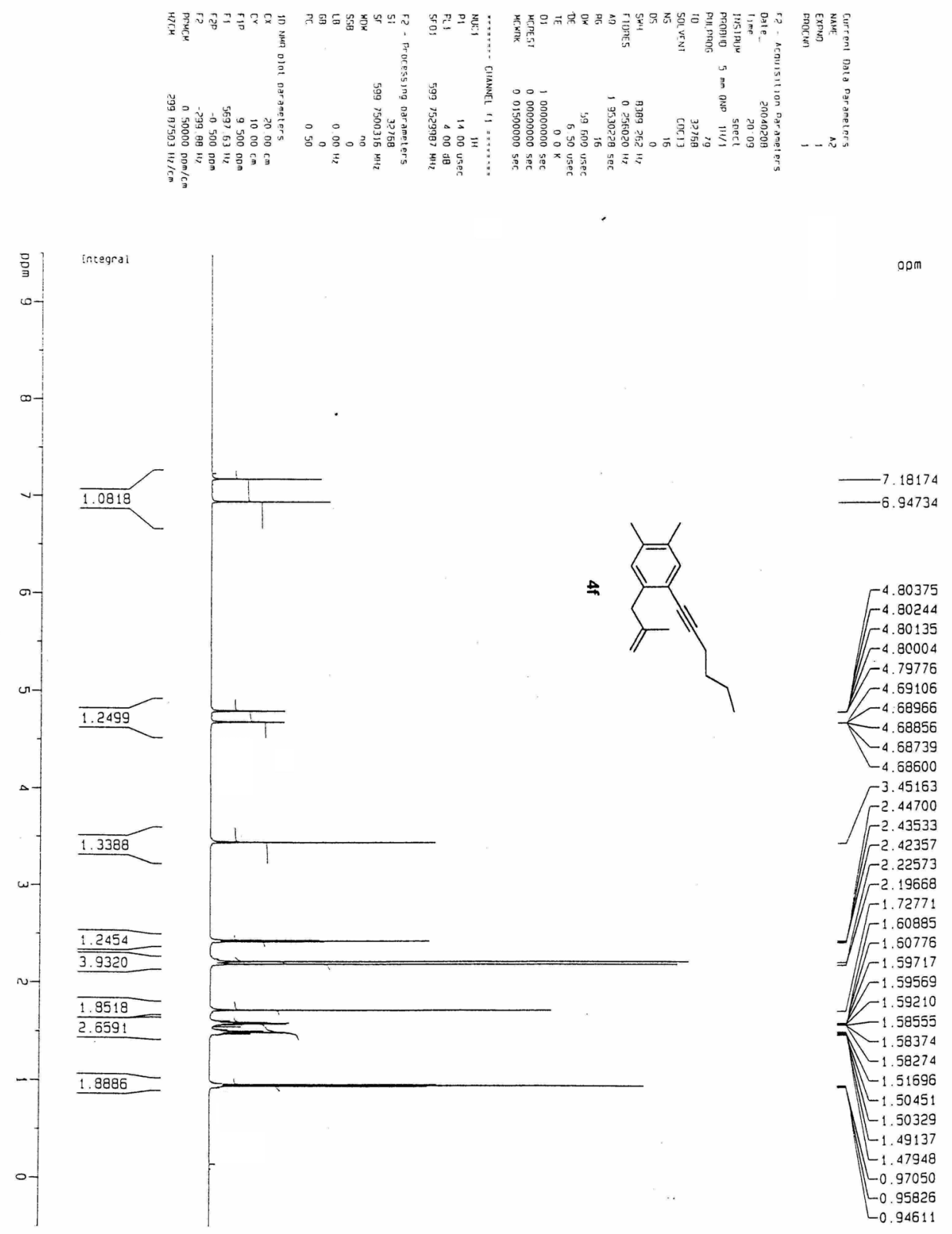

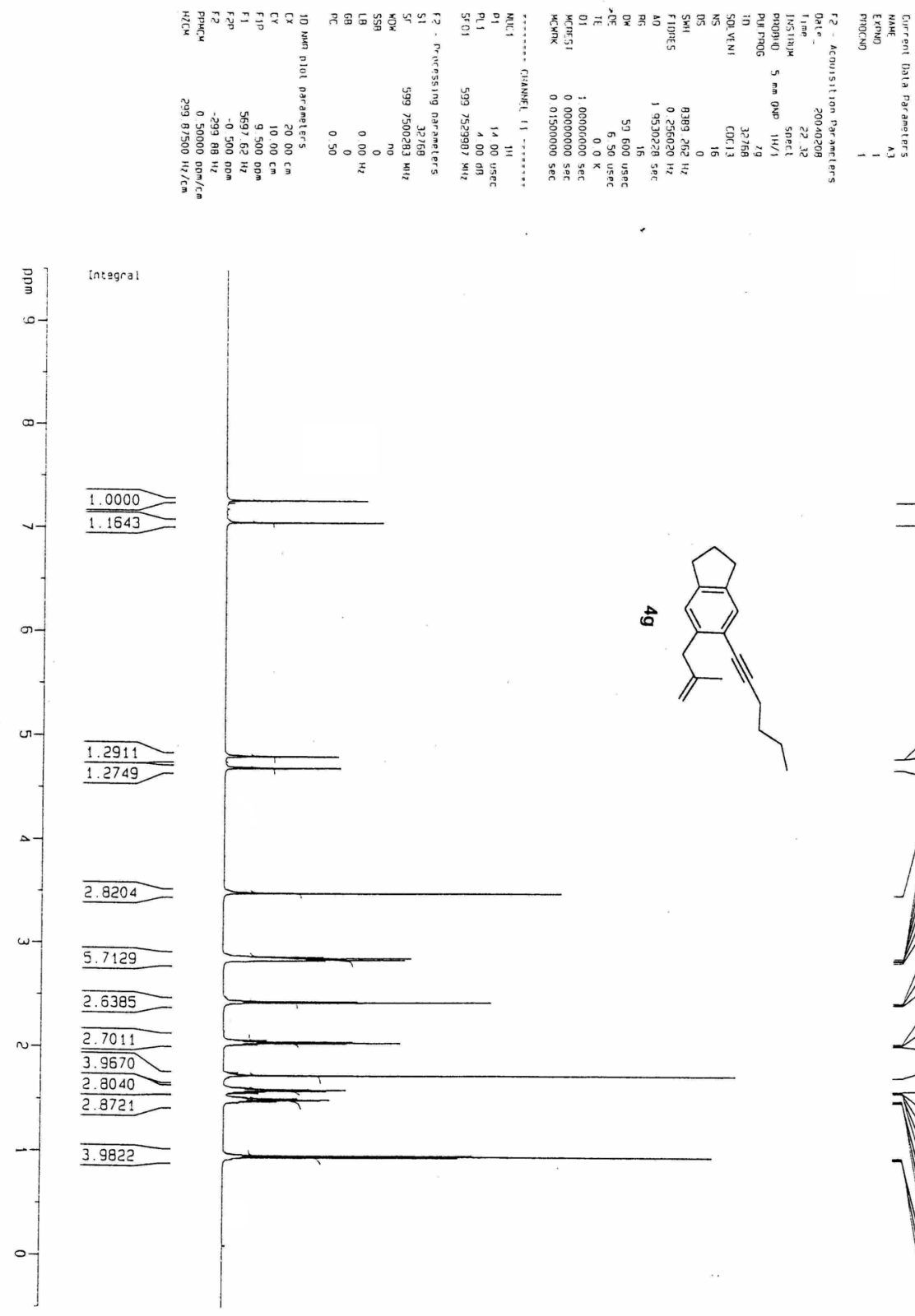

роm
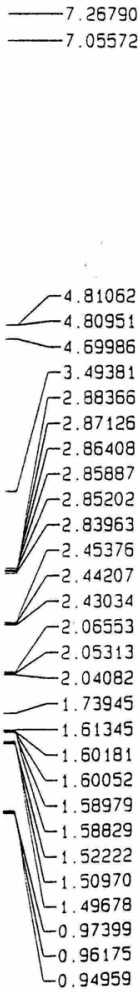


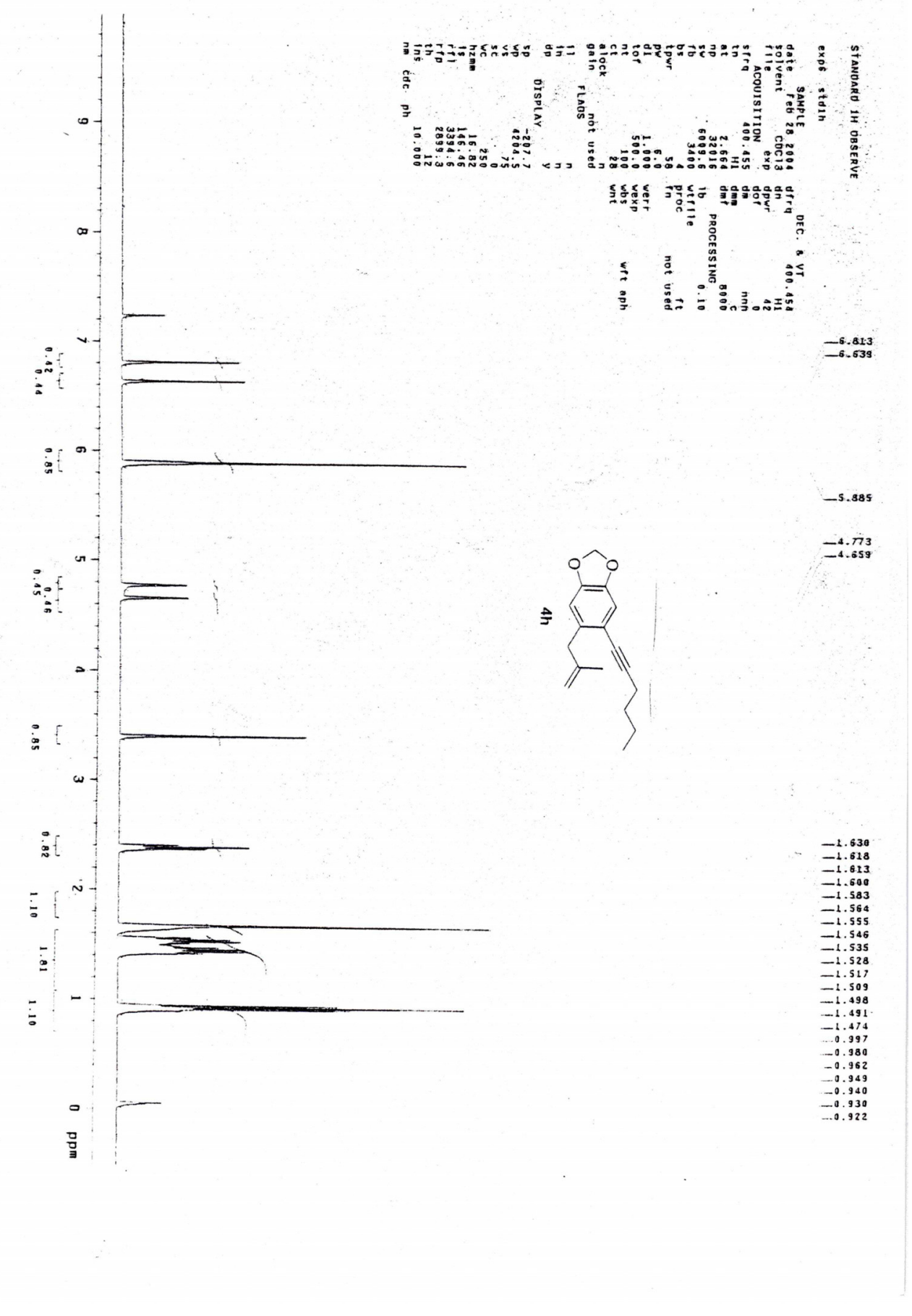




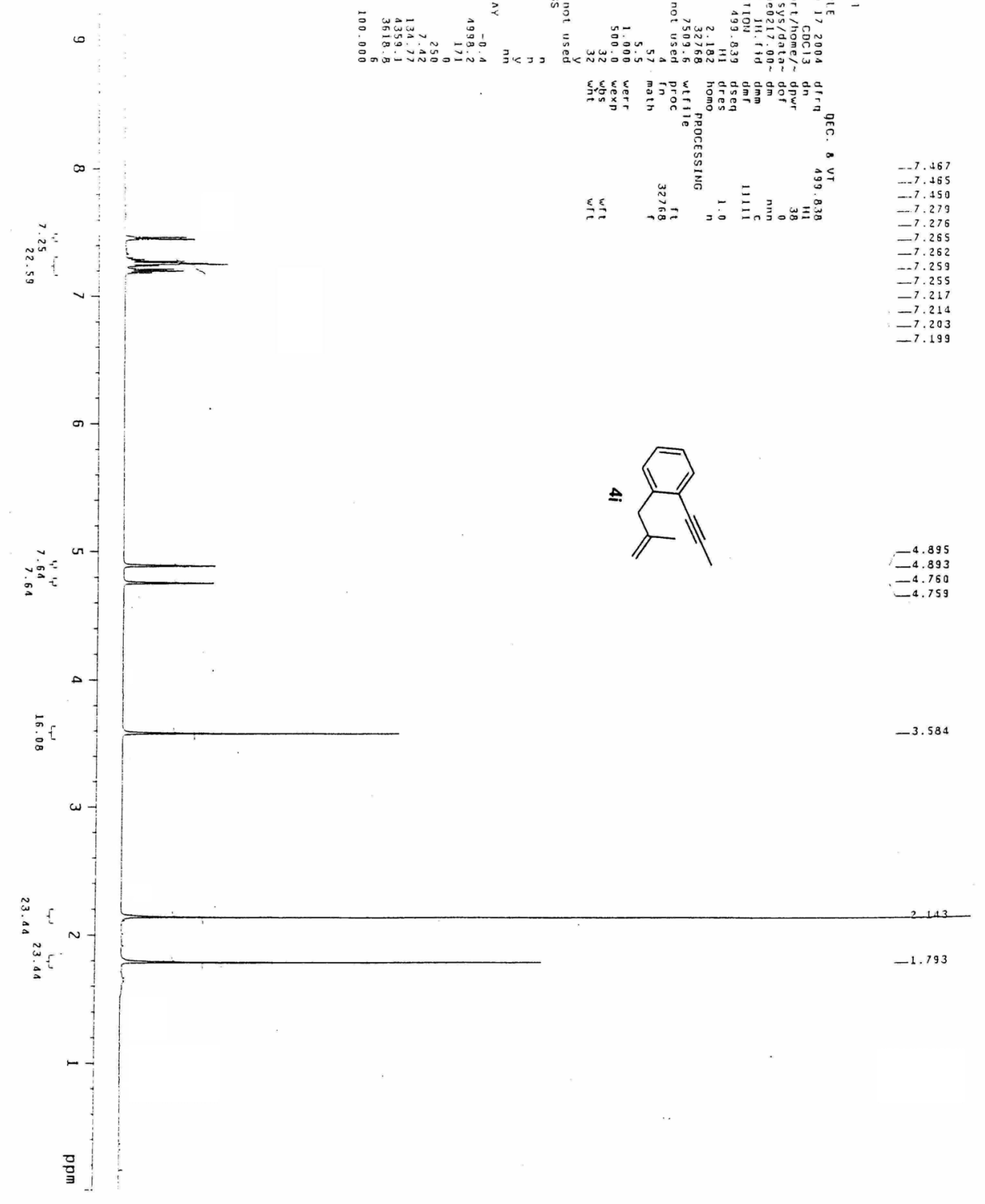




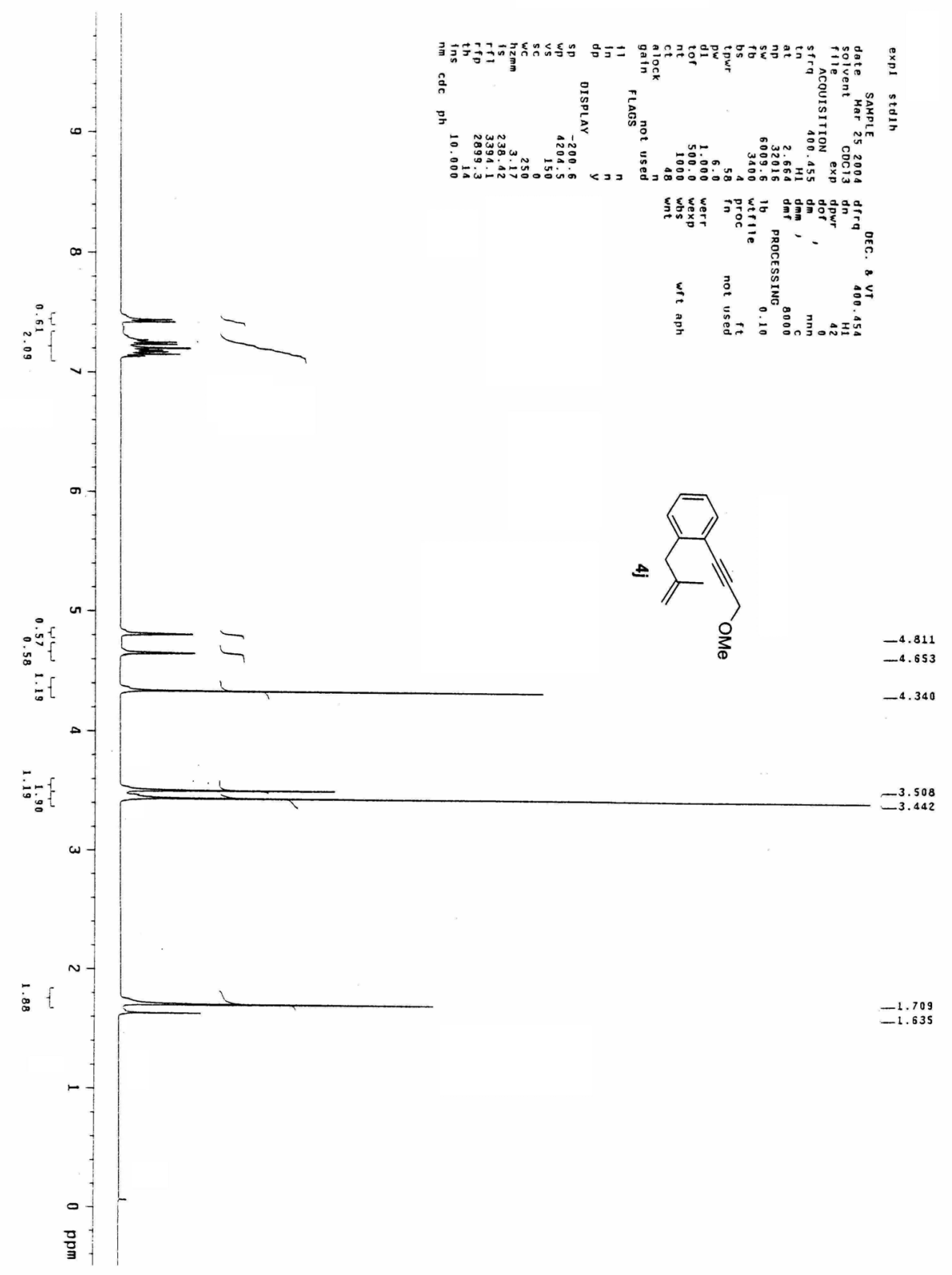



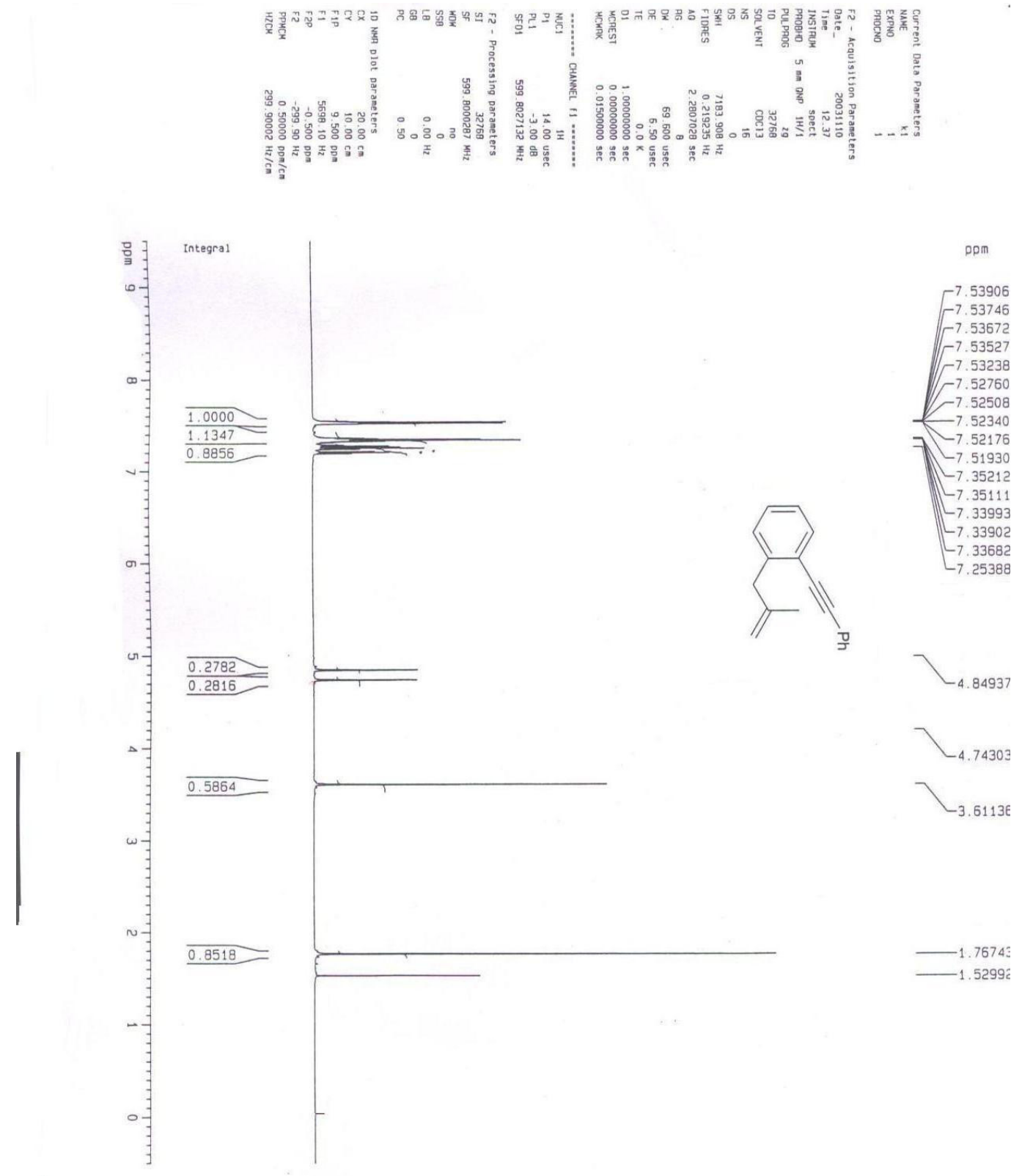

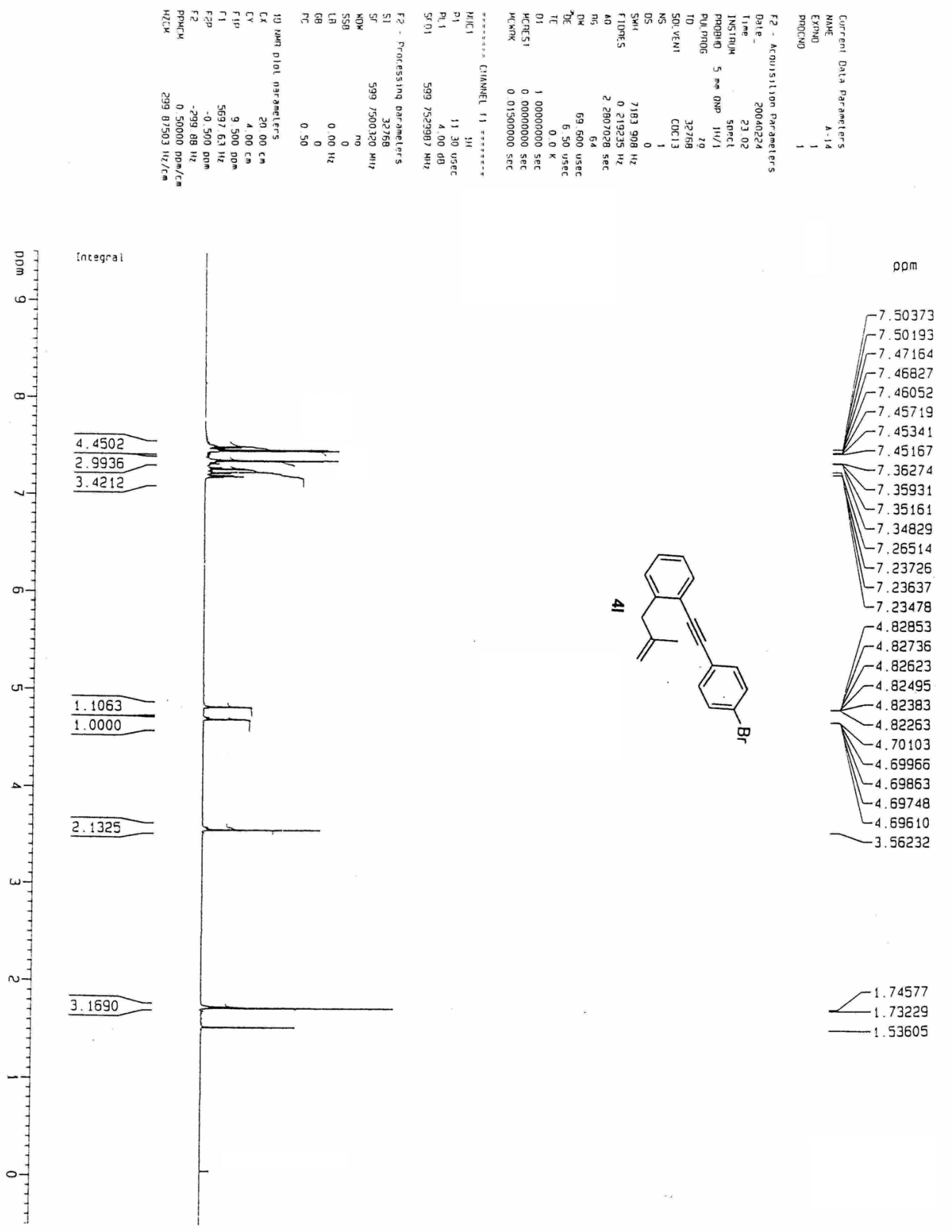

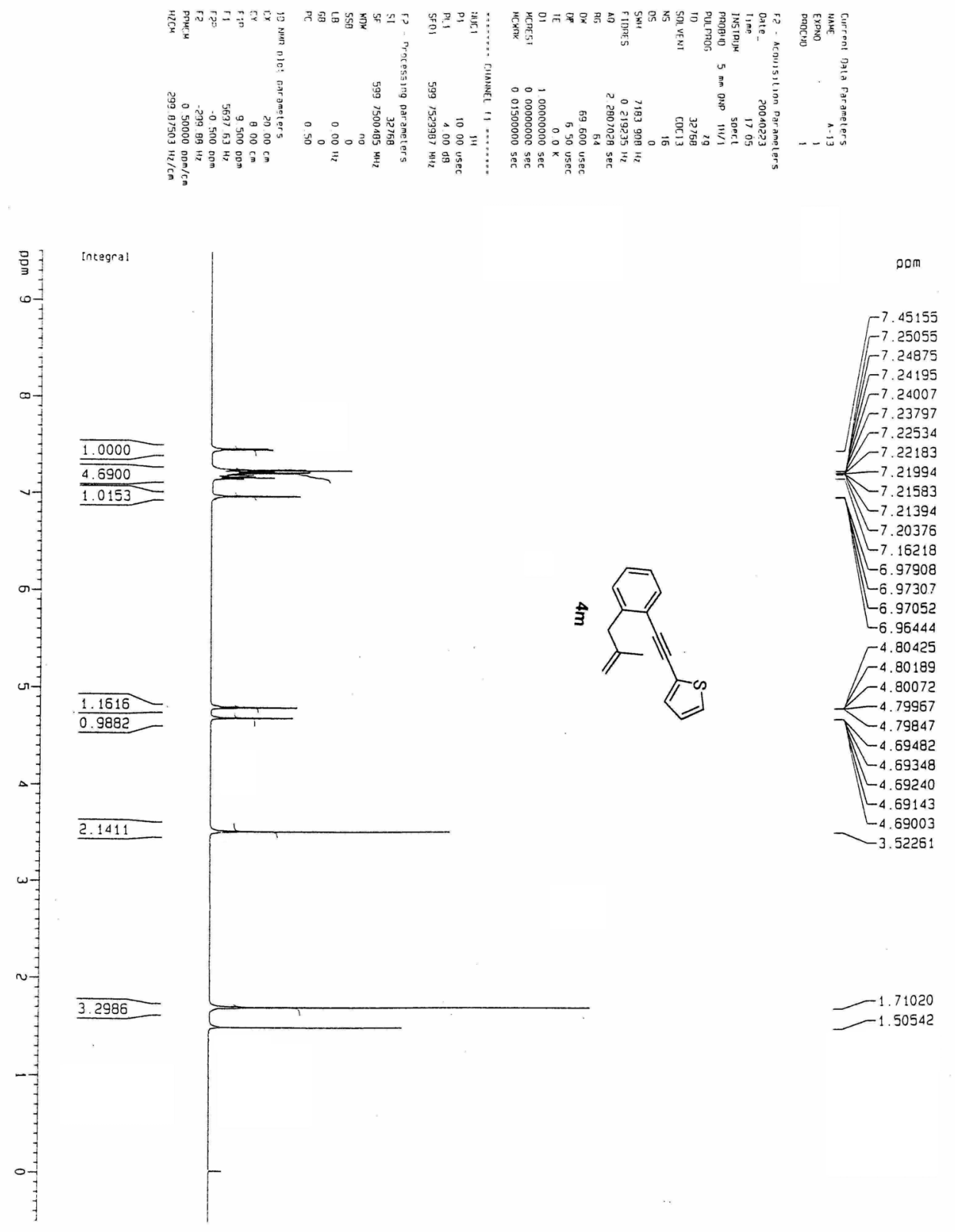

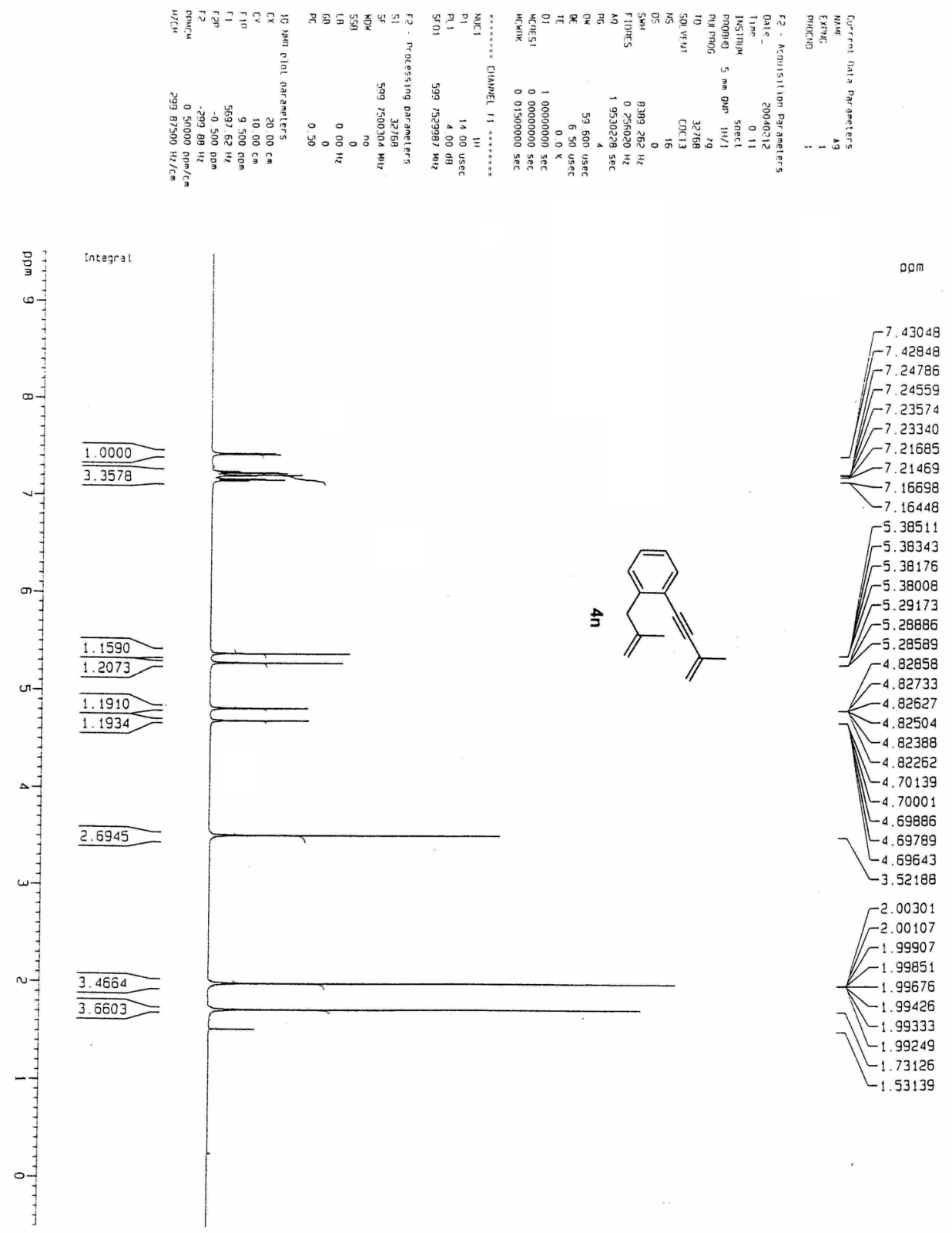

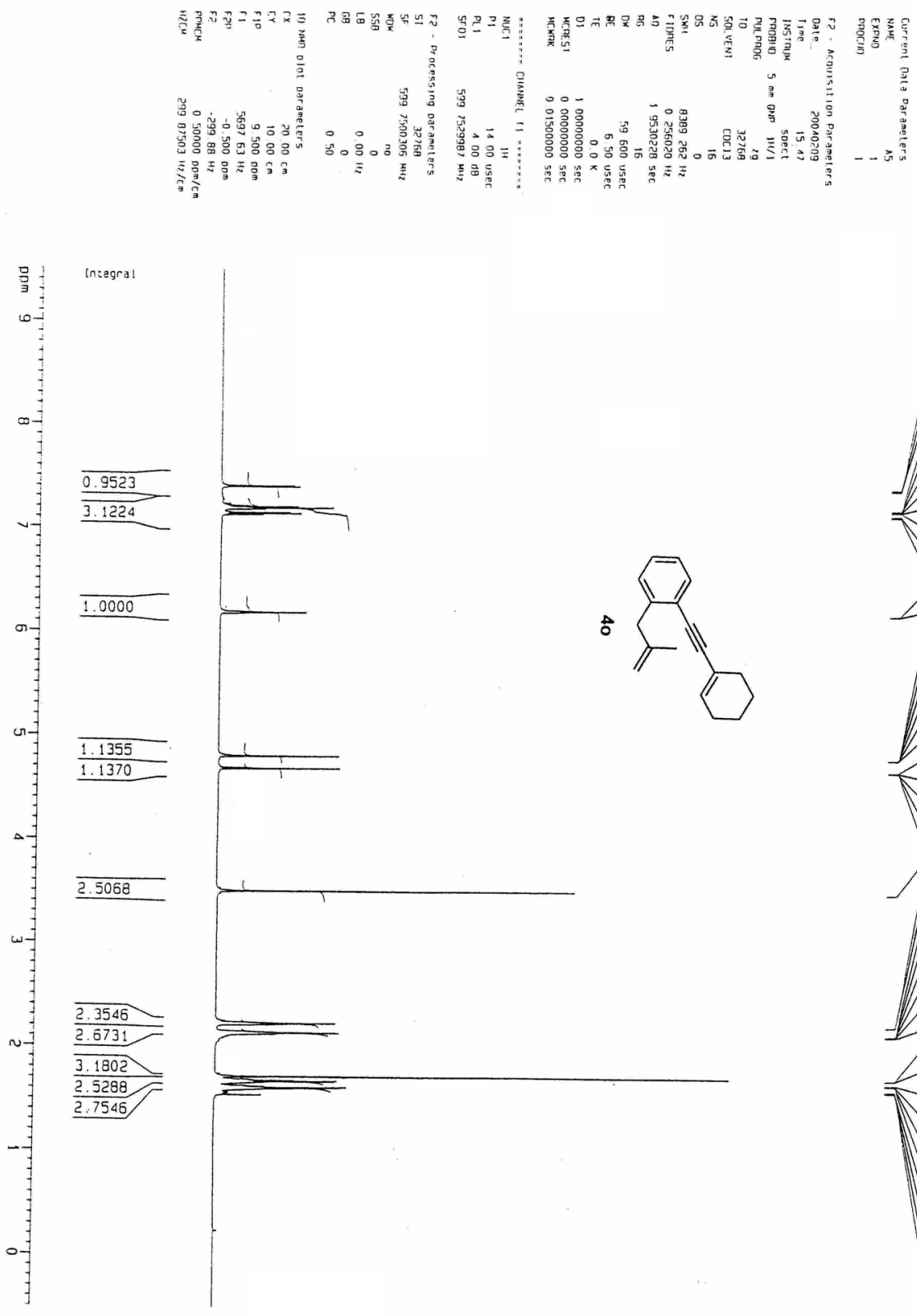

opm

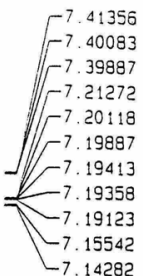

-6.19250

$-6.18932$

4.81270
4.81146

4.81027

$-4.80902$

$-4.80785$

$-4.80672$

4.69270

4.69123

$-4.69038$

$-4.68909$

4.68769

3.50983
2.23222

$-2.22882$

$-2.14782$

$-2.14382$

-2.14137

-2.13939

$\begin{array}{r}2.13709 \\ -2.13343 \\ \hline\end{array}$

$-1.72243$

1.72243
-1.72126

$-1.68263$

$\mathcal{L}_{-1.67890}$

$L_{1.67261}$

$-1.66895$

$L_{1.62165}$

$\left[\begin{array}{l}-62165 \\ -1.61797\end{array}\right.$

$L_{1.61797}$

$L_{1.61178}$ 

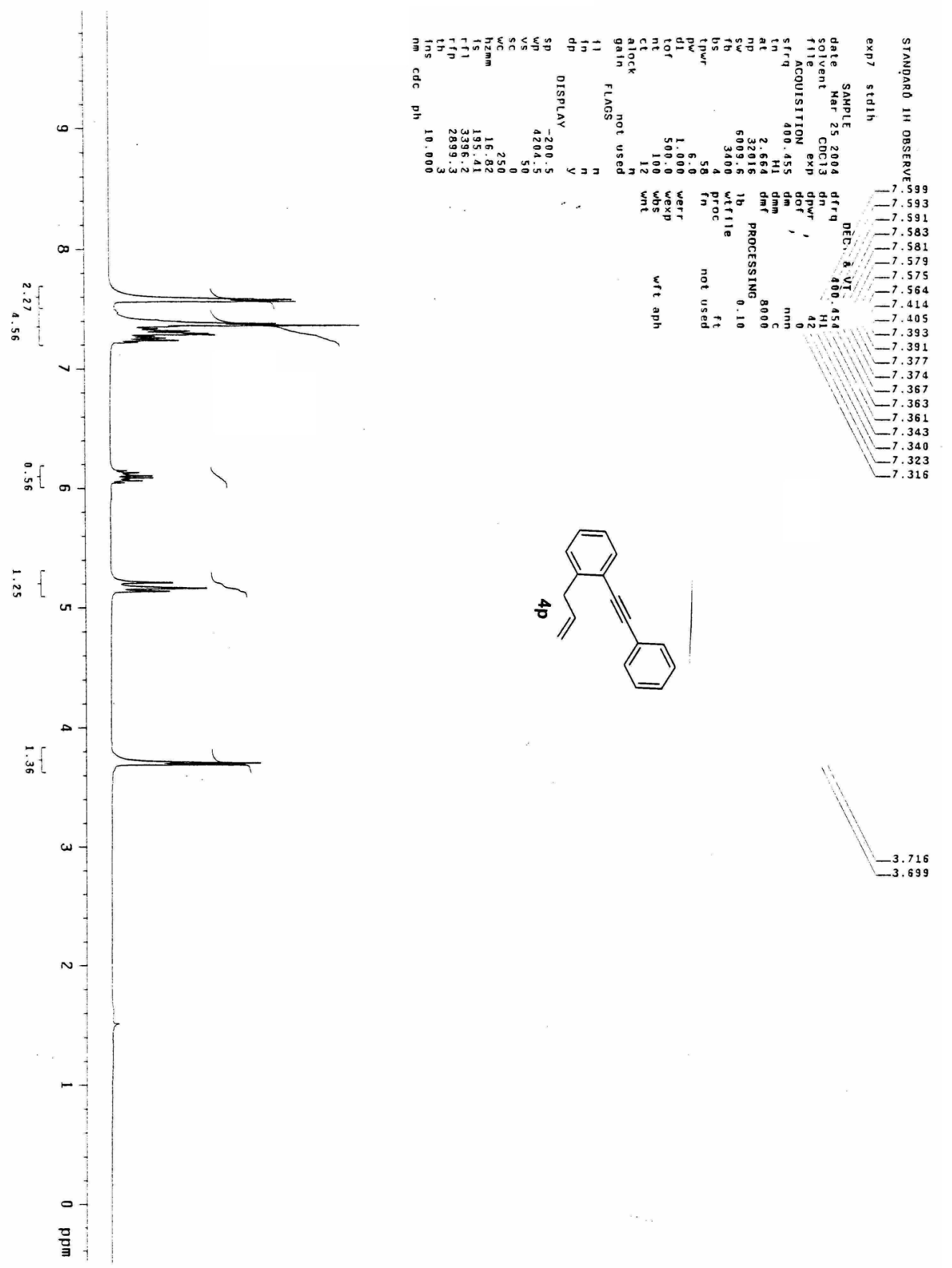


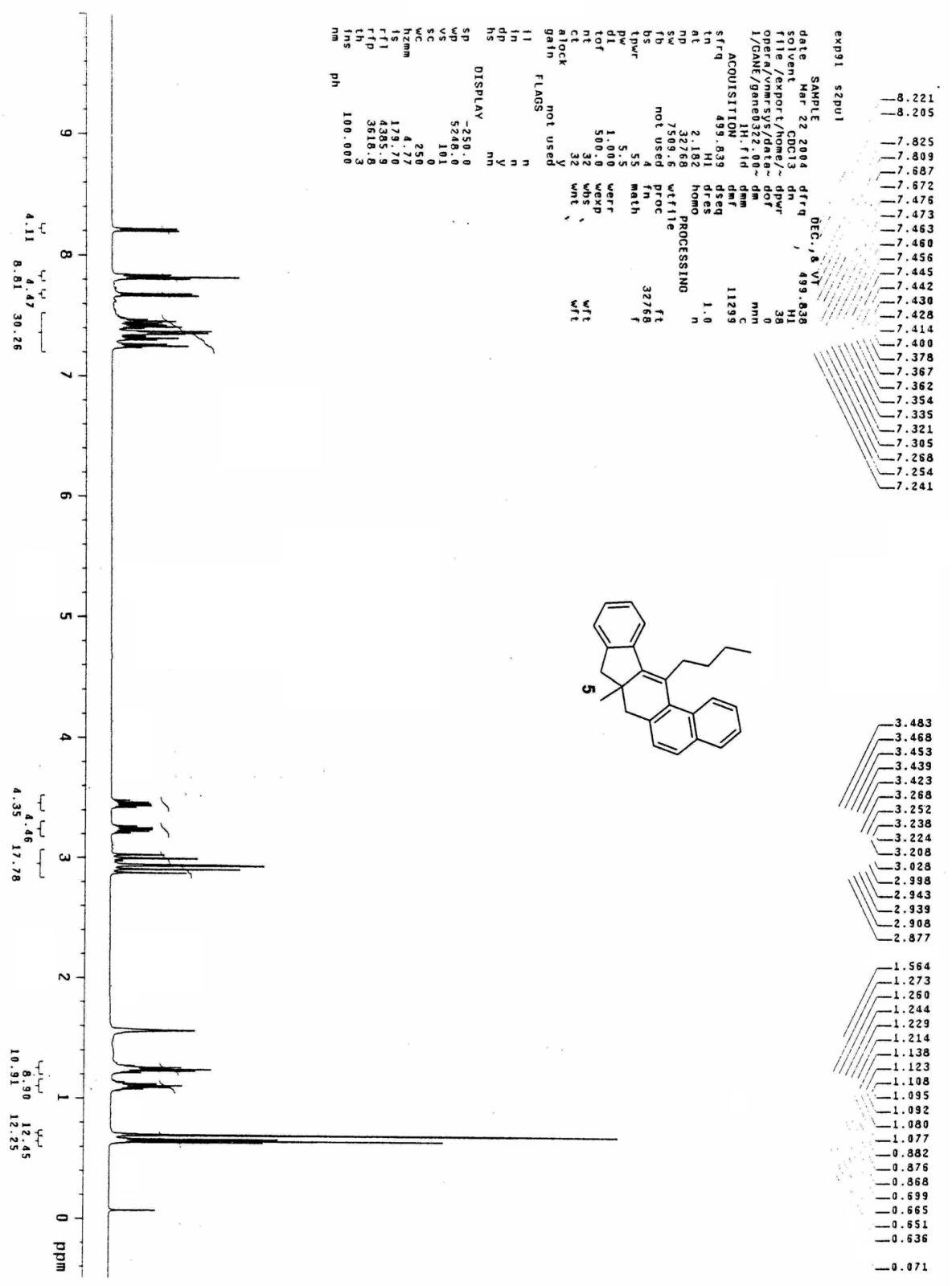



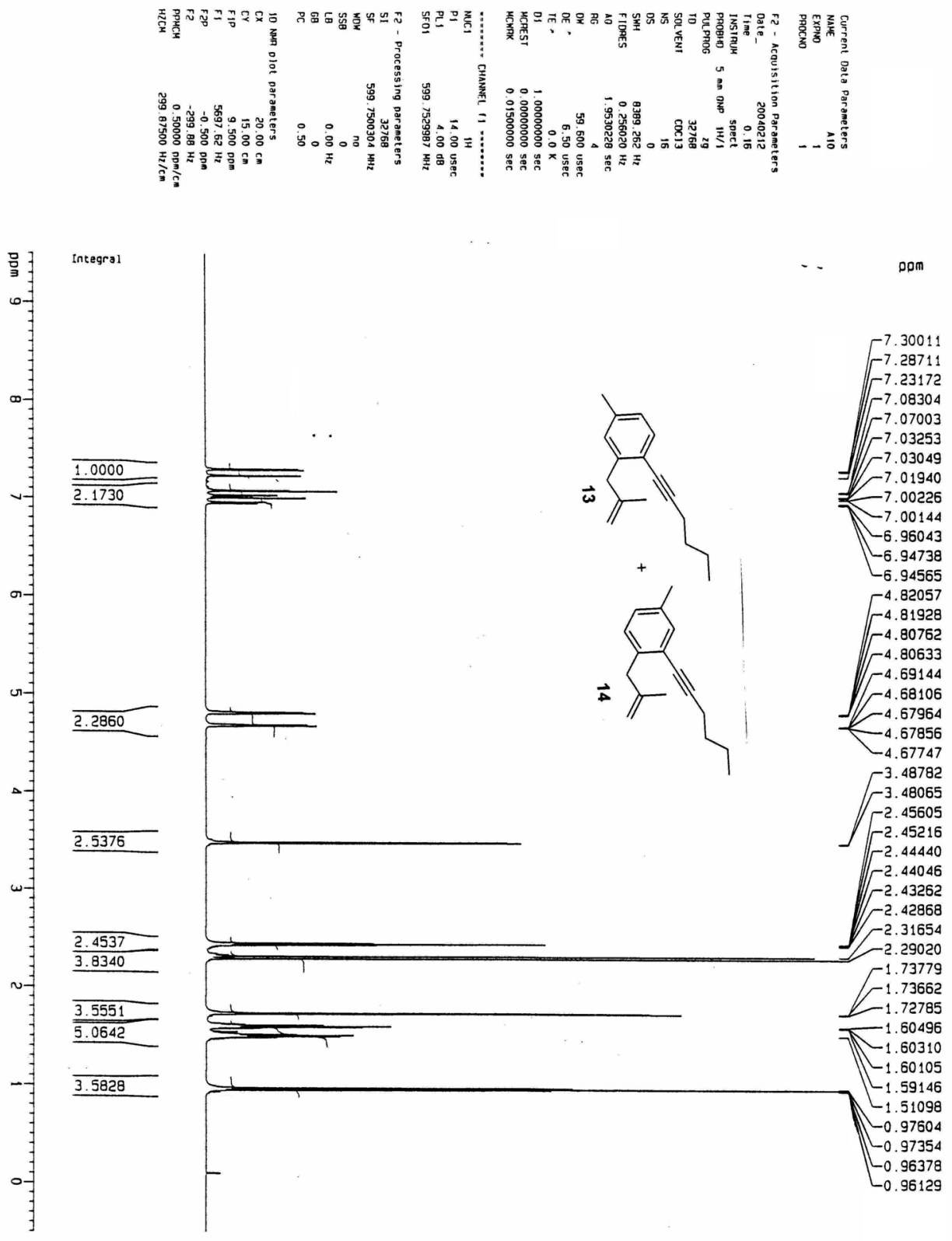